\title{
Hartree-Fock ground state of the two-dimensional electron gas with Rashba spin-orbit interaction
}

\author{
L. O. Juri and P. I. Tamborenea \\ Departamento de Física "J. J. Giambiagi", Universidad de Buenos Aires \\ Ciudad Universitaria, Pabellón I, C1428EHA Ciudad de Buenos Aires, Argentina \\ Submitted 26 February 2007, published in Phys. Rev. B 27 June 2008
}

\begin{abstract}
We search for the uniform Hartree-Fock ground state of the two-dimensional electron gas formed in semiconductor heterostructures including the Rashba spin-orbit interaction. We identify two competing quantum phases: a ferromagnetic one with partial spin polarization in the perpendicular direction and a paramagnetic one with in-plane spin. We present a phase diagram in terms of the relative strengths of the Rashba to the Coulomb interaction and the electron density. We compare our theoretical description with existing experimental results obtained in GaAs-AlGaAs heterostructures.
\end{abstract}

PACS numbers: 71.10.Ca, 71.70.Ej, 73.21.-b

\section{INTRODUCTION}

The two-dimensional electron gas (2DEG) is a paradigmatic system of semiconductor physics and technology. Traditionally, the electronic spin degree of freedom in this system has played a secondary role. This situation has changed recently with the emergence of the promising field of spintronics. 1,2 The most controllable and often predominant spin-orbit coupling in semiconductor 2DEGs is the Rashba interaction. ${ }^{3,4}$ It is then important to determine the various many-body properties of the 2DEG in its presence. The ground state of the uniform 2DEG without spin-orbit interaction is not known exactly ${ }_{5,6,7}$ In this Brief Report we concentrate on how the single-particle Rashba term affects the ground state in the Hartree-Fock (HF) mean-field approximation. $\frac{8}{-}$ While for the uniform electron gas without spin-dependent potentials, the HF approach yields trivial single-particle spin-orbitals (plane waves and pure spin states), the presence of the Rashba spin-orbit interaction causes the HF solution to possess an intriguing spin texture in momentum space. In this Brief Report we formulate the HF theory and obtain solutions for the uniform 2DEG with Rashba interaction. Our main finding is a spatially uniform ferromagnetic phase, characterized by a net out-of-plane partial magnetization appearing in a window of densities. $\frac{9}{}$

\section{HARTREE-FOCK THEORY WITH RASHBA SPIN-ORBIT INTERACTION}

The Hamiltonian of the 2DEG in the presence of the Rashba spin-orbit interaction is

$$
H=\sum_{i} H_{R, i}+\frac{1}{2} \sum_{i \neq j} v\left(\mathbf{r}_{i}-\mathbf{r}_{j}\right) .
$$

The second term gives the interparticle Coulomb interaction $v\left(\mathbf{r}_{i}-\mathbf{r}_{j}\right)=e^{2} / \varepsilon\left|\mathbf{r}_{i}-\mathbf{r}_{j}\right|$, where $\varepsilon$ is the dielectric constant of the semiconductor, and the first term is the sum of the Rashba Hamiltonians of the individual electrons. The latter are given by

$$
H_{R}=-\frac{\hbar^{2} \nabla^{2}}{2 m^{*}}-i \alpha\left\langle E_{z}\right\rangle\left(\sigma_{x} \frac{\partial}{\partial y}-\sigma_{y} \frac{\partial}{\partial x}\right)
$$

Here, $m^{*}$ is the conduction-band effective mass, $\alpha\left\langle E_{z}\right\rangle$ is a structural parameter that determines the strength of the Rashba coupling, and $\sigma_{x}$ and $\sigma_{y}$ are Pauli matrices. The Rashba Hamiltonian can be solved analytically, and the following wave functions and energies are obtained: 3

$$
\begin{gathered}
\psi_{\mathbf{k} s}(\mathbf{r})=\frac{1}{\sqrt{2 A}} e^{i \mathbf{k} \cdot \mathbf{r}}\left(\begin{array}{c}
i s e^{-i \varphi} \\
1
\end{array}\right), \\
E(k, s)=\frac{\hbar^{2} k^{2}}{2 m^{*}}+s \alpha\left\langle E_{z}\right\rangle k .
\end{gathered}
$$

In these expressions $\mathbf{k}$ is the two-dimensional wave vector, $\varphi$ is the angle of $\mathbf{k}$ in polar coordinates, and $A$ is the surface area of the sample. The spin quantum number $s= \pm 1$ denotes spin-up and spin-down eigenstates with respect to the spin quantization axis, which lies in the $\mathrm{x}-\mathrm{y}$ plane and is perpendicular to $\mathbf{k}$ with a polar angle $\phi_{R}(\mathbf{k})=\varphi-\pi / 2$. Notice that, however, the Rashba ground state is paramagnetic. $\stackrel{4}{*}$ Also, the Rashba Hamiltonian $H_{R}$ is time-reversal invariant. This invariance requires that $\psi_{\mathbf{k} s}(\mathbf{r})$ and $\psi_{-\mathbf{k} s}(\mathbf{r})$ be Kramers-conjugate states with the same energy eigenvalue $E(k, s)$ (Ref. 10).

For a spatially uniform solution the HF spin-orbitals can be written as

$$
\psi_{\mathbf{k} s}(\mathbf{r})=\frac{1}{\sqrt{2 A}} e^{i \mathbf{k} \cdot \mathbf{r}} \chi(\mathbf{k}, s) .
$$

The spinor $\chi(\mathbf{k}, s)$ has components $\chi_{ \pm}(\mathbf{k}, s)$, which are the unknown spinor amplitudes to be determined through the HF procedure. Notice that while the Rashba amplitudes of Eq. (3) depend only on the polar angle $\varphi$ of $\mathbf{k}$, we allow $\chi_{ \pm}(\mathbf{k}, s)$ to depend also on the modulus of k. The spin quantum number $s= \pm 1$ denotes, like in the 
noninteracting Rashba problem, the up- and down-spin eigenstates in an unknown spin-quantization axis $\hat{\mathbf{u}}(\mathbf{k})$ with polar angles $\theta(\mathbf{k})$ and $\phi(\mathbf{k})$.

The functional that one has to minimize is given by (direct Coulomb terms drop out in the jellium-model electron gas)

$$
\begin{aligned}
& \mathcal{F}=\sum_{s, i}^{N_{s}} \chi^{\dagger}\left(\mathbf{k}_{i}, s\right)\left[H_{R}\left(\mathbf{k}_{i}\right)-E\left(\mathbf{k}_{i}, s\right)\right] \chi\left(\mathbf{k}_{i}, s\right) \\
& -\frac{1}{2 A} \sum_{s s^{\prime}, i j}^{N_{s} N_{s^{\prime}}} v\left(\mathbf{k}_{i}-\mathbf{k}_{j}\right)\left[\chi^{\dagger}\left(\mathbf{k}_{j}, s^{\prime}\right) \chi\left(\mathbf{k}_{i}, s\right)\right] \\
& \times\left[\chi^{\dagger}\left(\mathbf{k}_{i}, s\right) \chi\left(\mathbf{k}_{j}, s^{\prime}\right)\right],
\end{aligned}
$$

where $N_{s}$ are the numbers of occupied orbitals with $s=$ $\pm 1, E\left(\mathbf{k}_{i}, s\right)$ are the HF single-particle energies, $v\left(\mathbf{k}_{i}-\right.$ $\left.\mathbf{k}_{j}\right)=2 \pi e^{2} / \varepsilon\left|\mathbf{k}_{i}-\mathbf{k}_{j}\right|$ is the Fourier transform of the Coulomb interaction, and

$$
H_{R}(\mathbf{k})=\left(\begin{array}{cc}
\hbar^{2} k^{2} / 2 m^{*} & i \alpha\left\langle E_{z}\right\rangle k e^{-i \varphi} \\
-i \alpha\left\langle E_{z}\right\rangle k e^{i \varphi} & \hbar^{2} k^{2} / 2 m^{*}
\end{array}\right) .
$$

We minimize this functional with respect to the amplitudes $\chi_{\epsilon}^{*}\left(\mathbf{k}_{i}, s\right)$ and obtain the single-particle energies,

$$
\begin{aligned}
& E(\mathbf{k}, s)=\frac{\hbar^{2} k^{2}}{2 m^{*}}+s \alpha\left\langle E_{z}\right\rangle k \sin \theta(\mathbf{k}) \\
& -\frac{1}{2 A} \sum_{s^{\prime}, \mathbf{k}^{\prime} \in \mathcal{D}_{s^{\prime}}} v\left(\mathbf{k}-\mathbf{k}^{\prime}\right)\left[1+s s^{\prime} \hat{\mathbf{u}}(\mathbf{k}) \cdot \hat{\mathbf{u}}\left(\mathbf{k}^{\prime}\right)\right] .
\end{aligned}
$$

By demanding that the single-particle HF Hamiltonian be diagonal on the basis of the spinor amplitudes $\chi_{\epsilon}\left(\mathbf{k}_{i}, \pm\right)$ we obtain the following integral equations:

$$
\begin{gathered}
2 \alpha\left\langle E_{z}\right\rangle k \sin [\phi(\mathbf{k})-\varphi] \cos \theta(\mathbf{k})= \\
\frac{1}{A} \sum_{s^{\prime}, \mathbf{k}^{\prime} \in \mathcal{D}_{s^{\prime}}} s^{\prime} v\left(\mathbf{k}-\mathbf{k}^{\prime}\right)\left\{\sin \theta(\mathbf{k}) \cos \theta\left(\mathbf{k}^{\prime}\right)\right. \\
\left.-\sin \theta\left(\mathbf{k}^{\prime}\right) \cos \theta(\mathbf{k}) \cos \left[\phi(\mathbf{k})-\phi\left(\mathbf{k}^{\prime}\right)\right]\right\}, \\
2 \alpha\left\langle E_{z}\right\rangle k \cos [\phi(\mathbf{k})-\varphi]=\frac{1}{A} \sum_{s^{\prime}, \mathbf{k}^{\prime} \in \mathcal{D}_{s^{\prime}}} s^{\prime} v\left(\mathbf{k}-\mathbf{k}^{\prime}\right) \\
\times \sin \theta\left(\mathbf{k}^{\prime}\right) \sin \left[\phi(\mathbf{k})-\phi\left(\mathbf{k}^{\prime}\right)\right] .
\end{gathered}
$$

In these equations, we have substituted the following expressions for $\chi_{\epsilon}\left(\mathbf{k}_{i}, \pm\right)$ in order to display the dependence on the polar angles $\theta(\mathbf{k})$ and $\phi(\mathbf{k}): \chi_{+}(\mathbf{k},+)=$ $\cos (\theta / 2) \exp (-i \phi / 2), \quad \chi_{-}(\mathbf{k},+)=\sin (\theta / 2) \exp (i \phi / 2)$, $\chi_{+}(\mathbf{k},-)=-\sin (\theta / 2) \exp (-i \phi / 2)$, and $\chi_{-}(\mathbf{k},-)=$ $\cos (\theta / 2) \exp (i \phi / 2)$. The summation domains $\mathcal{D}_{ \pm}$are the regions of $k$ space occupied by electrons and their areas are $N_{ \pm}$, respectively. Notice that Eq. (8) reduces to: (i) Eq. (44) when the Coulomb interaction is neglected because the third term of the right-hand side (RHS) of Eq. (8) drops out and $\theta(\mathbf{k})=\pi / 2$ in the single-particle Rashba problem; (ii) the HF one-particle energy if the Rashba coupling is omitted because $\alpha=0$ and $\hat{\mathbf{u}}(\mathbf{k}) \equiv \hat{\mathbf{z}}$.

\section{ISOTROPIC SOLUTION}

In the absence of Pomeranchuk instabilities 11 (PI) (deformations of the Fermi sphere in three dimensions or circle in two dimensions), the domains $\mathcal{D}_{ \pm}$should be taken as having circular symmetry. The issue of the occurrence of PI in Fermi liquids with isotropic central interactions is currently being studied in two-dimensional (2D) and three-dimensional (3D) systems $\underline{12,13}$ In the particular case of the bare Coulomb interaction (which is our case), the existing theory is not able to categorically predict or rule out the PI. However, a screened Coulomb interaction does not produce PI (Ref. 13), which can be taken as an indication that no PI occurs either for the bare interaction. In general, it is safe to assume that no PI will occur unless: (i) there is a well-defined length scale in the interaction, and (ii) that length scale is larger than the mean interparticle distance $\underline{14}$ These conditions are clearly not satisfied by the bare Coulomb interaction, which does not possess a characteristic length scale. Based on these results, it is safe to assume that our integration domains have circular symmetry.

The circular symmetry of the integration domains implies that the dispersion relations $E(\mathbf{k}, s)$ in Eq. (8) must be isotropic. This in turn, requires that $\theta(\mathbf{k})$ be independent of $\varphi$ and that $\phi(\mathbf{k})=\varphi-\pi / 2$ as in the noninteracting Rashba problem. ${ }^{15}$ Thus Eq. (10) is automatically satisfied and Eq. (9) becomes

$$
\begin{aligned}
& p x \cos \theta(x)=\int_{x_{c}}^{1} \int_{0}^{2 \pi} \frac{x^{\prime} d x^{\prime} d \varphi^{\prime}}{\sqrt{x^{2}+x^{\prime 2}-2 x x^{\prime} \cos \left(\varphi-\varphi^{\prime}\right)}} \\
& \times\left[\sin \theta(x) \cos \theta\left(x^{\prime}\right)-\cos \theta(x) \sin \theta\left(x^{\prime}\right) \cos \left(\varphi-\varphi^{\prime}\right)\right] \cdot(11)
\end{aligned}
$$

We have introduced the parameter $p=2 \alpha\left\langle E_{z}\right\rangle \varepsilon / e^{2}$ which indicates the relative strength of the Rashba and Coulomb interactions. The integration limit $x_{c}$ contains the information of the integration domains $\mathcal{D}_{ \pm}$ introduced earlier. Having in mind the dispersion relation of the noninteracting Rashba problem described in Eq. (4), the situation can be summarized as follows: when both branches are occupied (high density), the domains $\mathcal{D}_{ \pm}$are filled Fermi circles of radii $k_{F \pm}$ related by $4 \pi n_{s}=k_{F+}^{2}+k_{F-}^{2}$, with $n_{s}=\left(N_{+}+N_{-}\right) / A$. In this case $x_{c}=k_{F+} / k_{F-}$ and, in principle, it is free to vary from zero to one. If only the lower branch is occupied (low density), there are two possibilities. If the lower branch has a minimum at $k=0$ (unlike the noninteracting Rashba problem), then there is a gap at $k=0$ between the two branches. In this case we define $x_{c}$ as before, obtaining $x_{c}=0$. The formation of a gap is enabled by the lifting of Kramers degeneracy at the single-particle level, due to the appearance of a spontaneous magnetization. Kramers' theorem states that the degeneracy of half-integer spins can only be removed by a magnetic field; we return to this point in the discussion of Eq. (12). If the lower branch does have a minimum at $k \neq 0$ we take $x_{c}=k_{\min } / k_{\max }$, where $k_{\min }\left(k_{\max }\right)$ are the inner (outer) Fermi radii of the hollow circular 
domain, now being $4 \pi n_{s}=k_{\max }^{2}-k_{\min }^{2}$. In this case, there is no gap at $k=0$, no (ground-state) magnetic moment appears and the time-reversal symmetry (Kramers degeneracy) is preserved. In Eq. (11), $x=|\mathbf{x}| \equiv|\mathbf{k}| / k_{F-}$ or $x=|\mathbf{k}| / k_{\max }$, according to the context.

Clearly, the noninteracting Rashba states with $\theta=$ $\pi / 2$, are a solution of Eq. (11). We call this the in-plane (IP) paramagnetic phase, which is the one we mentioned as having the lower dispersion-relation branch with a minimum at $k \neq 0$. The nontrivial solution with varying $\theta(x)$ gives rise to an OP ferromagnetic phase where, in turn, the lower branch has its minimum at $k=0$. We solve Eq. (11) starting with the initial guess of $\theta_{0}(x)=0$, which gives $\theta_{1}(x)$ after integration. With $\theta_{1}(x)$ as an input, we obtain $\theta_{2}(x)$ and so on. We consider that convergence is achieved when $\theta_{n}(x)$ and $\theta_{n-1}(x)$ differ in less than 0.1 percent. Let us enumerate our main findings on $\theta(x)$ : (i) $\theta(0)=0$ for all values of $p$ (the Rashba spin-orbit interaction causes no effect when $k=0$ and consequently, the spin quantization axis must lie in the $z$ direction), (ii) $\theta(x)$ is a monotonically increasing function for all values of $p$, (iii) $\theta(x)$ increases with increasing $p$, and (iv) $\theta(x)$ never crosses the value $\pi / 2$.

The gap at $k=0$ between the two branches may be obtained from Eq. (8) by means of the above-mentioned properties, and it is given by

$$
\Delta E=E(0,+)-E(0,-)=\frac{e^{2} k_{F-}}{\varepsilon} \int_{x c}^{1} \cos \theta(x) d x .
$$

In the IP phase $\theta(x)=\pi / 2$ and then $\Delta E=0$. In the OP phase, in turn, we have $\Delta E \neq 0$. This gap, far from being a peculiarity of the OP phase, appears also in the HF theory of the 2DEG without Rashba coupling. In fact, setting $\theta(x)=0$ we get $\Delta E=\left(e^{2} / \varepsilon\right)\left(k_{F-}-k_{F+}\right)$ and the gap is unfailingly related to a polarized ground state, where $k_{F+} \neq k_{F-}$ (i.e., $N_{+} \neq N_{-}$) (an identical expression for $\Delta E$ multiplied by $2 / \pi$ can be obtained in a 3DEG: see the expression for $\epsilon_{k}^{ \pm}$when $k \rightarrow 0$ on p. 82 of Ref. 8).

\section{HARTREE-FOCK GROUND STATE}

The remaining task is, for given $p$ and $r_{s}\left(r_{s}=\right.$ $1 / a_{B}^{*} \sqrt{\pi n_{s}}$, where $a_{B}^{*}=\hbar^{2} \varepsilon / m^{*} e^{2}$ is the effective Bohr radius), to determine which of these two phases has lower energy. For each phase and given $r_{s}$, the value of $x_{c}$ that minimizes the energy is found numerically. In Fig. 1 we show a phase diagram in terms of the parameters $r_{s}$ and $p$. The striking feature of this diagram is that for given $0<p \lesssim 1.3$, the OP phase appears within a window of densities. As expected, for $p=0$ we recover the paramagnetic-ferromagnetic transition of the 2DEG HF approximation at $r_{s}=2.01$ (Refs. 16 and 17). As $p$ increases, the left transition moves slightly towards smaller $r_{s}$; in other words, the presence of the Rashba coupling favors a spin-polarized phase, albeit this polarization is partial for nonzero $p$ (the original HF ferro-

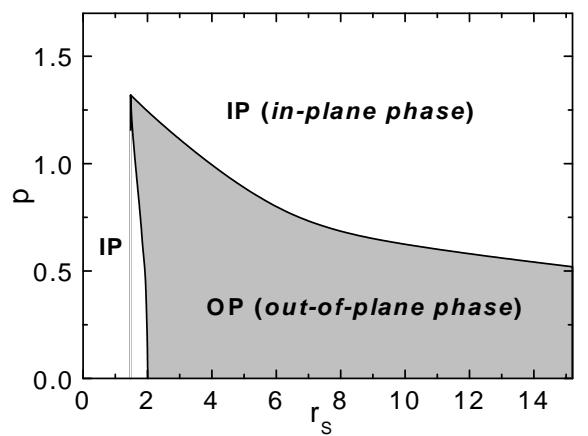

FIG. 1: Ground-state phase diagram in the Hartree-Fock approximation in terms of the density parameter $r_{s}$ and the Rashba to Coulomb energy ratio $p$.

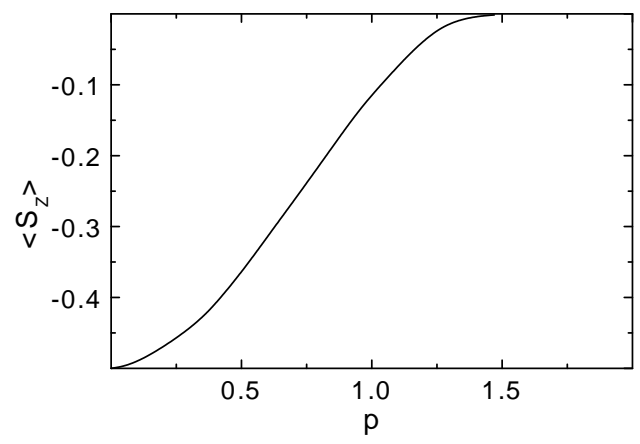

FIG. 2: Mean value of the perpendicular spin projection per particle $(\hbar=1)$ as a function of the Rashba to Coulomb energy ratio in the OP phase.

magnetic phase has full polarization). Also the window of densities in the OP phase shrinks as $p$ increases. The right transition originates in the fact that the system diminishes the ground-state energy by filling the lower branch that has minimum at $k \neq 0$ (IP phase), when $r_{s}$ increases at fixed $p$.

The partial spin polarization of the (ferromagnetic) OP phase can be seen in Fig. 2, where we plot the mean value of $S_{z}$ per particle $\left(\left\langle S_{x}\right\rangle=\left\langle S_{y}\right\rangle=0\right.$ for both IP and OP phases) given by $(\hbar=1)$,

$$
\left\langle S_{z}\right\rangle=-\int_{x_{c}=0}^{1} x \cos \theta(x) d x .
$$

Notice that $\left\langle S_{z}\right\rangle$ does not depend on $r_{s}$ since $x_{c}=0$ for the OP phase $\frac{18}{18}$

\section{COMPARISON WITH EXPERIMENT AND CONCLUSION}

In a recent experimental work, Ghosh et al. $\frac{19}{}$ report a possible spontaneous spin polarization in mesoscopic twodimensional systems. They studied 2DEGs in asymmetric $\mathrm{Si} \delta$-doped GaAs/AlGaAs heterostructures with den- 
sities as low as $n_{s}=5 \times 10^{9} \mathrm{~cm}^{-2}\left(r_{s}=7.6\right)$. The temperature was set at $T \approx 40 \mathrm{mK}$ or equivalently $T / T_{F} \approx 0.02$ since $T_{F}=2.3 \mathrm{~K}$ at $r_{s}=7.6$. According to their interpretation of the data, these authors found partial spin polarization estimated as $\zeta \equiv\left(N_{+}-N_{-}\right) / N \approx 0.2$ appearing in a window of densities of width $\Delta r_{s} \approx 1.8$, centered around $r_{s} \approx 6.5$. After a detailed analysis of their data, the authors rule out the Rashba coupling and invoke an exchange-driven spontaneous spin polarization in order to explain their observed split zero-bias peak (ZBP) in the differential conductance. On the other hand, they explain the fact that the spin polarization is partial as a finite-temperature effect. Recall that at zero temperature, this transition is of first order and is predicted to occur between $r_{s} \approx 13$ (Ref. 5) and $r_{s} \approx 25$ (Ref. 7). However, this contradicts the theoretical finding of Dharma-wardana and Perrot, ${ }^{20}$ in the sense that partial spin polarization due to the exchange happens for $T / T_{F}$ between 0.3 and 1.6, i.e., well above $T / T_{F} \approx 0.02$ as reported by Ghosh et al.

Our theory supports the interpretation of the experiment of Ghosh et al. based on an exchange-driven spinpolarized ground state. On the other hand, we explain the partial spin polarization in terms of the Rashba interaction rather than in terms of finite temperature. This hypothesis could be tested by additional experiments with a tunable Rashba coupling, which would allow varying our parameter $p$. In such experiments the degree of spin polarization could be modified and measured.

Ghosh et al. observe a split ZBP within a window of electron densities. At the lower end of this window, disorder-induced localization destroys the electron-gas scenario. In a defect-free sample, we also predict a disappearance of the split ZBP but due entirely to the competition between Rashba and exchange interactions $\stackrel{21}{=}$ At the higher end of the density window, the reason for the disappearance of the split ZBP is not as clear, and it is not specified by the authors. According to our theory, this feature could be interpreted as the ferromagneticparamagnetic transition of the electron gas at high density, seen in Fig. 1 for low $r_{s}$. (Of course, the critical value of $r_{s}$ is overestimated by the Hartree-Fock approximation because it leaves out many-body correlations. ${ }^{22}$ )

In summary, we have found the uniform Hartree-Fock ground state of a 2DEG in the presence of spin-orbit Rashba coupling, characteristic of asymmetric semiconductor quantum wells. We present a phase diagram where two competing quantum phases are identified, one of which shares the Rashba single-particle orbitals, with their IP spin quantization axis, and another one which has a finite component of the spin in the perpendicular direction. This phase possesses a partial spin polarization and exists within a window of densities, suggesting that a combination of exchange and Rashba spin-orbit interaction may qualitatively explain experimental results obtained in GaAs-AlGaAs heterostructures at low electron density.

\section{Acknowledgments}

We thank J. Quintanilla for his useful comments. We acknowledge financial support from UBACyT, CONICET and ANPCyT. P.I.T. is a researcher of CONICET.
1 Semiconductor Spintronics and Quantum Computation, edited by D. D. Awschalom, D. Loss, and N. Samarth (Springer, Berlin, 2002).

2 I. Zutic, J. Fabian, and S. Das Sarma, Rev. Mod. Phys. 76, 323 (2004).

3 E. I. Rashba, Sov. Phys. Solid State 2, 1109 (1960). Y. A. Bychkov and E. I. Rashba, JETP Lett. 39, 78 (1984); J. Phys. C 17, 6039 (1984).

4 R. Winkler, Spin-Orbit Coupling Effects in TwoDimensional Electron and Hole Systems (Springer, Berlin, 2003).

5 D. Ceperley, Phys. Rev. B 18, 3126 (1978).

${ }^{6}$ G. Ortiz, M. Harris, and P. Ballone, Phys. Rev. Lett. 82, 5317 (1999).

7 C. Attaccalite, S. Moroni, P. Gori-Giorgi, and G. B. Bachelet, Phys. Rev. Lett. 88, 256601 (2002).

8 E. K. U. Gross, E. Runge, and O. Heinonen, Many-Particle Theory (Hilger, Bristol, 1991).

9 We learned after submission of our manuscript that results similar to the ones presented here have been reported in S. Chesi, G. Simion, and G. F. Giuliani, arXiv:cond-mat/0702060 (unpublished).

10 E. I. Rashba, Phys. Rev. B 68, 241315(R) (2003).

11 I. Ia. Pomeranchuk, Sov. Phys. JETP 35, 524 (1958).
12 J. Quintanilla, C. Hooley, B. J. Powell, A. J. Schofield and M. Haque, Physica B 403, 1279 (2008).

13 J. Quintanilla and A. J. Schofield, Phys. Rev. B 74, 115126 (2006).

14 J. Quintanilla (private communication).

15 More precisely, the circular symmetry of the dispersion relation requires that $\phi(\mathbf{k})=\phi_{0}(k)+n \varphi$ where $n$ is an integer and $\phi_{0}(k)$ is an arbitrary function. However, Eq. (10) determines that $n=1$ and analyzing the expression for the HF ground-state energy (not shown), we realize that it is a minimum when $\phi_{0}(k)=\phi_{0}=$ const. Finally, Eq. (10) forces $\phi_{0}= \pm \pi / 2$, and as in the single-particle Rashba problem, we choose $N_{-}$as the majority number, i.e., $\phi_{0}=-\pi / 2$.

16 R. J. Radtke, P. I. Tamborenea, and S. Das Sarma, Phys. Rev. B 54, 13832 (1996).

17 A. K. Rajagopal and J. C. Kimball, Phys. Rev. B 15, 2819 (1977).

18 The $r_{s}$ dependence of $x_{c}$ may be summarized as follows: (i) in the OP phase $x_{c}=0$ because only the lower branch is occupied, (ii) in the IP phase at high density, on the contrary, for given $p, x_{c}$ decreases monotonically as $r_{s}$ increases due to the occupancy of both branches, and (iii) $x_{c}$ increases monotonically in the IP phase at low density 
since only the lower branch is occupied and $k_{\min } \rightarrow k_{\max }$ when $r_{s}$ increases.

19 A. Ghosh, C. J. B. Ford, M. Pepper, H. E. Beere, and D. A. Ritchie, Phys. Rev. Lett. 92, 116601 (2004).

20 M. W. C. Dharma-wardana and F. Perrot, Phys. Rev. Lett. 90, 136601 (2003).

21 Thus, localization at low density anticipates and masks the OP-IP transition seen in Fig. 1 Note from Fig. 2 that $\zeta \approx 0.2$ corresponds to $p \approx 1$ and with this value we get, from Fig. 1] a window of densities $\Delta r_{s} \approx 2$.

22 L. O. Juri and P. I. Tamborenea, Eur. Phys. J. B 45, 9 (2005). 